\title{
Percepción de la identidad corporativa de una institución de educación superior
}

\author{
Recibido: 13 de agosto de 2018 \\ Aceptado: 1 de octubre de 2018 \\ Publicado: 28 de noviembre de 2018
}

\author{
Yolanda López Lara \\ yolanda.lopezlr@uanl.edu.mx \\ Universidad Autónoma de Nuevo León (México)
}

Resumen: El objetivo general del presente artículo es determinar si el Sistema de Identidad Corporativa (SIC) de la Universidad Autónoma de Nuevo León (UANL) transmite elementos de notoriedad de dependencia a sus públicos internos. Asimismo, se indaga si la acción sinérgica de los signos que conforman el SIC aumenta su eficacia en conjunto y, además, se busca definir si los públicos internos se apropian de los signos lingüísticos, icónicos y cromáticos del SIC.

Las tendencias (que se obtuvieron de las encuestas aplicadas) aportaron datos de diagnóstico sobre la apropiación de los signos de la identidad corporativa por parte de la comunidad estudiantil, brindando la oportunidad de que dichos resultados se difundan y así crear expectativas de oferta académica y de intercambio a públicos externos de otras universidades a nivel nacional e internacional, manteniéndose la atención de los stakeholders. También permite hacer recomendaciones de mejora para el programa de Relaciones Públicas Internas de la Facultad de Ciencias de la Comunicación (FCC) y para la Dirección de Intercambio Académico (DIA) de la UANL.

Palabras clave: Identidad corporativa, institución de educación superior, docentes, relaciones públicas, reputación.

\begin{abstract}
The main aim of this paper is to determine if the Corporate Identity System (SIC) of Universidad Autónoma de Nuevo León (UANL) transmits notoriety elements of dependence to its internal audiences. Likewise, this research analyzes if synergic action of signs, that make up the SIC, increases its effectiveness as a whole. Furthermore, this paper defines if internal publics appropriate linguistic, iconic and chromatic SIC signs.
\end{abstract}

The trends (obtained from surveys) provide diagnostic data about signs appropriation of corporate identity by university students. This provides the opportunity for these results 
to be disseminated and thus create expectations for academic offer and exchange with external publics from other universities (domestic and foreign), keeping stakeholders attention. It also allows recommendations for improvement for Internal Public Relations program of Faculty of Communication Sciences (FCC) and for UANL Academic Exchange Office (DIA).

Key words: Corporate Identity, Higher Education Institution, Teachers, Public Relations, Reputation.

\section{Introducción}

En la actualidad, muchas organizaciones procuran mantener una imagen corporativa positiva ante los ojos de la comunidad. Por ese motivo, emplean diferentes estrategias para comunicar y posicionarse en la mente del público objetivo. Cuando se hace referencia a la identidad corporativa, estas se remiten a los elementos distintivos que les otorgan singularidad.

Las organizaciones (desde su planeación) son construidas con los elementos particulares que formarán su "personalidad", tales como su nomenclatura social o comercial; misión, visión, filosofía; logotipo y colores, entre otros rubros que la identifican en su entorno y de la competencia. La suma de estos elementos conforma una identidad corporativa (IC) a la institución y las operaciones (que sus líderes desarrollan estratégicamente) permiten que los públicos externos (clientes, proveedores, mercados, competidores, autoridades, comunidad, entre otros) perciban al organismo con una carga emocional particular, según sea el contexto en que se le posicione.

La UANL, consciente de su identidad y del papel que desarrolla en la formación académica en el nivel medio-superior y superior (en la zona noreste de México) creó e implementó un extenso programa de internacionalización, contemplado en el "Plan de Desarrollo Visión 2020-UANL", en el que suscribe (como eje prioritario y transversal) el impulso y consolidación de los procesos de internacionalización, con el fin de posicionar a la UANL como una institución educativa de clase mundial, reconocida por su compromiso de coadyuvar en la formación de sus egresados al conferirles capacidades sustanciales para competir a nivel global.

Dentro de su estructura orgánica, la UANL cuenta con el Centro de Internacionalización, cuya función principal es establecer los lazos con instituciones y organismos extranjeros, promoviendo el desarrollo de una formación científica humanista y proyectos de investigación. En su diseño organizacional, coordina con la DIA en cada una de las 26 facultades, siguiendo los lineamientos del programa central de intercambio y movilidad académica (no solo para estudiantes, sino también para profesores), por medio de los programas de relaciones públicas y el proceso de acompañamiento a los estudiantes y docentes durante el período que dure el intercambio académico en una universidad receptora. Además este centro es 
la carta de presentación de la Institución ante representaciones de autonomías extranjeras y de llevar a cabo las gestiones necesarias para la formalización de convenios de Cooperación Internacional y la ejecución de compromisos de movilidad y de liderazgo participativo en programas educativos internacionales (UANL, 2018b).

La Coordinación de Intercambio Académico de la FCC (cuya función principal es gestionar el programa de Relaciones Públicas y acompañamiento de los procesos de movilidad académica de profesores y estudiantes en el área de Comunicación, Periodismo y Mercadotecnia) se ubica dentro del organigrama de la Secretaría de Servicios Escolares y trabaja conjuntamente con un área de mayor representatividad a nivel institucional.

El estudio está enfocado en recolectar información de su público más cercano: los estudiantes. Anteriormente, la FCC brindaba la oferta de un solo programa académico (Licenciatura en Ciencias de la Comunicación). Al crearse dos programas académicos con un enfoque específico (la licenciatura en Periodismo Multimedia y la de Mercadotecnia y Gestión de la Imagen), atrajeron dos nuevos perfiles de públicos internos diferentes al anterior y que, a la postre, impactan el contexto de la comunidad estudiantil en actitudes y percepciones diferentes, pero complementarias.

\section{Comunicación en las organizaciones}

Robbins \& Judge (2017) mencionan que la comunicación tiene cinco funciones dentro de una organización: “administración, retroalimentación, expresión emocional, persuasión e intercambio" (p. 345). El proceso comunicativo actúa de diferentes formas para controlar el comportamiento de los miembros en base a lineamientos como las políticas, reglas y normas entre otros, que imperan en cada nivel jerárquico, según lo imponga la estructura organizacional, tomando en cuenta sus dimensiones, como son la formalización, la especialización, la jerarquía de autoridad, la centralización, el profesionalismo y los indicadores de recursos humanos (Benjamín \& Fincowsky, 2014, p. 4).

Al fomentarse la retroalimentación, se aclaran las funciones que deben realizar las diferentes áreas, que tan bien lo están haciendo y cómo podrían mejorar su rendimiento. La comunicación que se desarrolla dentro del grupo de trabajo es la principal fuente de interacción social que les permite expresar sus frustraciones, así como también sus satisfacciones en el desempeño de sus funciones.

Al igual que en la expresión de las emociones, la persuasión sería positiva o negativa, dependiendo del tipo de liderazgo que se compromete con la responsabilidad social. Un proceso de comunicación eficiente sigue los canales oficiales y los flujos de comunicación establecidos por su estructura organizacional, con la finalidad de transmitir mensajes relacionados con las actividades específicas a sus puestos y responsabilidades (Martínez de Velazco \& Nosnik, 2008). 


\subsection{Teoría de sistemas y comunicación}

El biólogo Ludwig Von Bertalanffy fundó las bases de la denominada "teoría general de los sistemas". Con un enfoque interdisciplinario, su objetivo es guiar la interacción y el desarrollo ordenado de las ciencias. Posteriormente este conocimiento fue adoptado por la teoría organizacional y revolucionó la perspectiva del estudio de las instituciones, convirtiendo al entorno externo en el elemento básico del formato y del comportamiento institucional (Chiavenato, 2009, p. 80).

Figura 1: La organización como un sistema abierto.

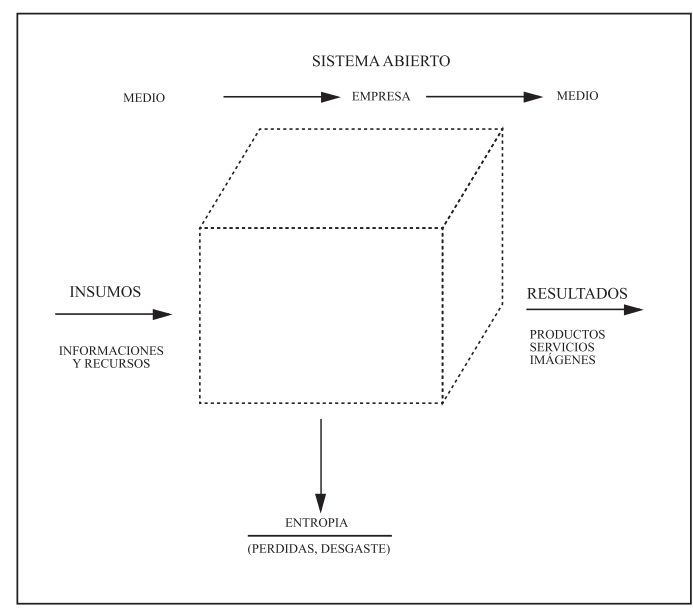

Fuente: Mello (2009, p. 13).

Una empresa organizada no existe en el vacío, sino que depende de su entorno externo y forma parte de sistemas mayores, como el sector industrial (al que pertenece), el sistema económico (que prevalece en su región geográfica) y la sociedad, donde nació con una serie de variables culturales que impactan en el desarrollo y desempeño de sus productos o servicios (Koontz, Weihrich \& Cannice, 2012, p. 27).

Katz \& Kahn, citados por Fernández (2014), logran sintetizar muchas de las ideas centrales de esta corriente, porque consideran a las organizaciones como un sistema abierto y viviente (figura 1), cuyas características principales son las entradas, transformación y salida y los subsistemas que lo conforman son los de producción, mantenimiento de la estructura, subsistemas de frontera o de soporte de la producción, adaptativos y gerenciales o administrativos (figura 2). Consideraban que las organizaciones funcionaban de manera interdependiente con su entorno (p. 29). 
Figura 2: Principales subsistemas y sus dimensiones.

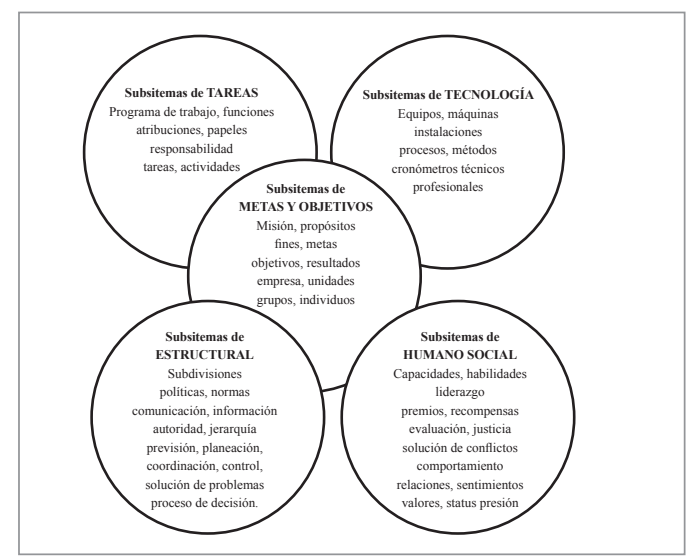

Fuente: Mello (2009, p. 19).

La importancia que esta teoría le concede a la comunicación es especialmente importante, porque la información es una "entrada" fundamental para el sistema de cualquiera de las partes. Asimismo, la retroalimentación es una constante como aspecto determinante en el control, la homeostasis y, en última instancia, el progreso del sistema. La función de la comunicación toma un papel central, por considerarse un elemento clave en la función de mantener los subsistemas interdependientes, interconectados e intercomunicados al constituir una totalidad. Las características de la comunicación en esta teoría se pueden visualizar en:

- La modalidad formal e informal entre el sistema y los subsistemas,

- En las formas oral y escrita,

- $\quad$ En la dirección de los mensajes, a nivel horizontal y vertical y transversal.

- Su regulación es variable, trata contenidos básicos relacionados en el ámbito organizacional interno y externo, además de ser cíclicos, repetitivos.

- Facilita la descentralización en forma variable, buscando el equilibrio interno y externo.

- Le da gran importancia a la retroalimentación como elemento central de control.

- Se consideran las necesidades de los individuos, los subsistemas y los sistemas.

- A nivel individual considera subsistemas y sistemas con énfasis en información de entrada (importada del medio) y salida, como elemento terminado (Fernández, 2014).

Un sistema abierto como la FCC implica que exista un intercambio de información entre el sistema universitario y su entorno interno y externo, lo cual significa que se encuentren en permanente interacción con el medio ambiente. Este intercambio o interacción es de tal naturaleza que logra mantener un equilibrio continuo. Las relaciones de la FCC con el entorno son tales que admiten cambios y adaptaciones al medio, conforme a su estructura y personalidad institucional (López Lara, 2010, p. 14). 


\subsection{Identidad corporativa}

Cuando se habla de identidad, por lo regular se alude a los rasgos de personalidad y carácter que los individuos tienen como atributos que los distinguen de otros y les proporcionan características específicas que los hacen únicos e irrepetibles. Estos mismos rasgos (transferidos al ámbito organizacional) conforman y le brindan una identidad corporativa que las singularizan entre muchas otras empresas del mismo ramo y de la competencia.

Todas las organizaciones, desde su constitución legal, están compuestas de varios elementos, tales como la nomenclatura social/comercial, el logotipo, colores corporativos, tipografía, marcas, productos y/o servicios, procesos, misión, visión, filosofía, valores, políticas, reglas, y normas, entre otros, según sea el enfoque de su giro, productos o servicio; elementos que las hacen distar de otras que se encuentran en el ámbito de la competencia (Costa, 2011).

La identidad corporativa debe ser considerada como un sistema de signos visuales cuyo objeto es diferenciar, acceder al reconocimiento y la remembranza de una organización, es la totalidad de los modos en que la organización se presenta a sí misma. Es la representación conceptual que el público tiene de ella, establecida por los aspectos visuales de la misma. Involucra la cantidad de información y de rasgos que distinguen a algo o a alguien evidenciando lo que es, y es dinámica y nace de tres elementos: “a) la historia de la empresa, b) su situación actual definida por el proyecto empresarial y c) la cultura corporativa" (Limón, 2008, p. 29).

En la opinión de Costa (2011), los signos que integran el SIC desempeñan una función similar, pero cada uno posee atributos comunicacionales diferentes. La noción de sistema implica la organización estructurada de los signos complementándose entre sí, con lo que provocan una acción sinérgica que aumenta su eficiencia en conjunto y consigue el efecto de constancia en la memoria del público. La IC está formada por signos de diferente naturaleza:

- $\quad$ Lingüística: el nombre de la empresa es un elemento de nominación verbal que el diseñador convierte en una grafía diferente. Un modo de escritura exclusiva llamada "logotipo".

- Icónica: se refiere a la marca gráfica o distintivo figurativo de la empresa, cuyo uso proviene de la costumbre ancestral del acto de marcar con una seña indeleble los objetos y las cosas (como los alfareros anteriores a Cristo marcaban sus producciones)

- Cromática: consiste en el color, o los colores que la empresa adopta como distintivo emblemático (Costa, 2011, pp. 15-16).

Por este acopio y remembranza en la memoria de los públicos, la IC excede sus funciones adyacentes y se convierte en un valor, es decir, en una imagen que constituye una base de comercio de la empresa, el cual representa uno de los principales activos de esta. 


\subsection{Identidad e identificación}

Toda organización tiene un conjunto de características que la distinguen y que permiten reconocerla diferenciándola de las demás. Estas cualidades específicas establecen su identidad, entendiéndola como la expresión de su propio ser (lo que existe).

De acuerdo con el diccionario de la Real Academia Española, la identidad se define como "calidad de idéntico. 2.f. Conjunto de rasgos propios de un individuo o de una colectividad que los caracteriza frente a los demás. 3.f. Conciencia de que una persona tiene de ser en ella misma y distinta a los demás. 4. f. Hecho de ser alguien o algo el mismo que se supone o se busca" (RAE, 2018). Estos mismos atributos se pueden considerar para una organización, tomándose en cuenta las características físicas, los aspectos mercadológicos y los elementos de la cultura organizacional.

Costa (2001) define la identificación como "el acto de reconocer la identidad de un sujeto, es decir, el acto de registrar y memorizar de modo inequívoco aquello que lo hace intrínsecamente diferente de todos los demás y al mismo tiempo idéntico a sí mismo (noción de unicidad)" (p. 16).

Figura 3: Modelo de representación de la identidad y la identificación.

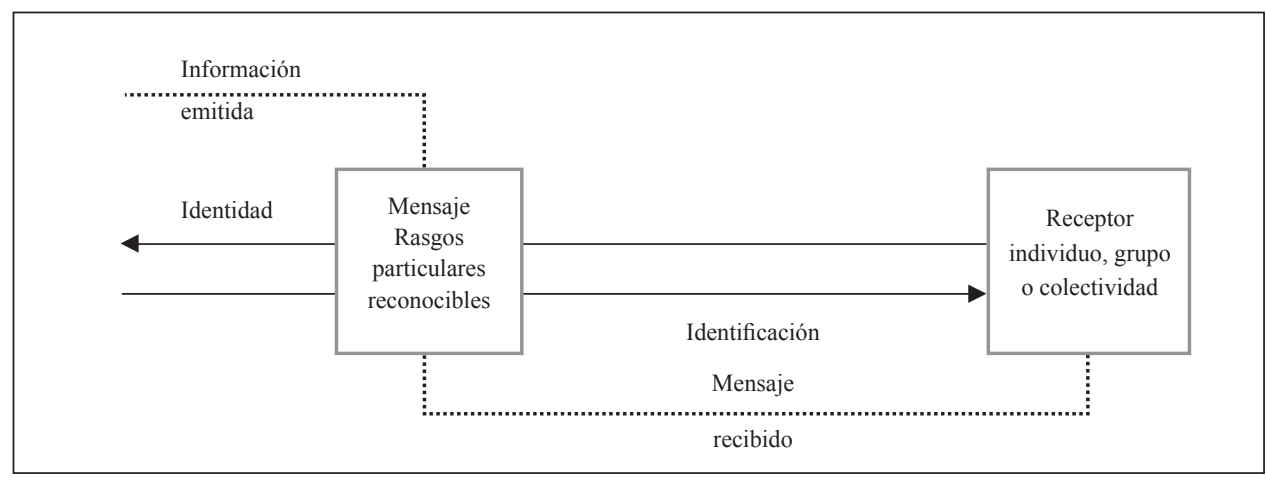

Fuente: Costa (2011, p. 17).

\section{Relaciones públicas}

Long \& Hazelton, citados por Wilcox, Cameron y Xifra (2012), las describen como "una función directiva de comunicación a través de la cual las empresas se adaptan, alteran o mantienen su entorno con el propósito de logrear las metas de la organización, donde las relaciones públicas son algo más que simple persuasión. También deberían fomentar una comunicación abierta, bilateral y una comprensión mutua, con la idea de que la empresa también cambie sus actitudes y comportamientos a lo largo del proceso, sin que esto se deje únicamente al público objetivo" (p. 7). 
Cameron, citado por Wilcox, Cameron y Xifra (2012), define esta disciplina como "la dirección estratégica de la competitividad y los conflictos en beneficio del titular de la organización (y cuando sea posible) también en beneficio mutuo tanto de la empresa como de sus diferentes púbicos" (p. 6).

Ríos (2006) explica que la función de relaciones públicas "es el conjunto de actividades efectuadas por cualquier organización para la creación y mantenimiento de buenas relaciones de los miembros la empresa y entre ésta y los demás sectores de la opinión pública, o 'públicos', tales como proveedores, clientes, inversionistas, gobierno y público en general, a fin de proyectar ente ellos una imagen favorable de la organización que contribuya al alcance de los objetivos de esta" (p. 13).

Marston (1999) las identifica como "la comunicación planificada y persuasiva destinada a influir sobre un público considerable” (p. 3). Cutlip \& Center, citados por Limón (2008), sostienen que "la función de las relaciones públicas es el esfuerzo planeado para influir en la opinión, por medio de la actuación aceptable y de comunicación recíproca" (p. 88). Asimismo, Ríos (2006) fundamenta que la gestión de las relaciones públicas son un esfuerzo de vincular a las empresas con sus públicos tomando en cuenta: “ $a$ ) la investigación, b) la elaboración de planes y programas generales o alternativos y c) la elaboración de planes y programas específicos" (p. 93).

Las relaciones públicas tienen un alto grado de importancia dentro de las empresas. El buen desempeño de estas conservan buenas relaciones de la empresa con sus públicos mediante la comunicación. En algunas organizaciones, las consideran una función de comunicación dentro de las mismas, motivo por el cual en la actualidad suelen llamarlas "comunicación empresarial" entre otros términos que resultan equívocos. Se les puede considerar como "un conjunto de acciones de comunicación y persuasión que tienen como objetivo influir en la opinión pública, para fortificar las relaciones con los distintos públicos, para lograr aprobación, fidelidad y sustento de los mismos en acciones a corto y largo plazo” (Limón, 2008, p. 87).

\subsection{Funciones de las relaciones públicas}

El departamento de relaciones públicas aborda una variedad de áreas administrativas interesadas, a nivel interno y externo. Todos los receptores de comunicación de la empresa son importantes. Por ese motivo, los mensajes deben ser claros y unificados, según el público al cual se dirigen. Con respecto a los públicos internos, los accionistas y el sindicato (así como el personal de la empresa) deben recibir un flujo constante de información. Para comunicarse eficazmente con los empleados, el departamento de relaciones públicas debe de trabajar con el departamento de recursos humanos. Por lo tanto, las publicaciones y comunicados que se difunden deben ser congruentes con la imagen y el mensaje que la empresa transmite a clientes y otros grupos.

En el caso de los públicos externos, la supervisión de la comunicación externa es una tarea compleja, porque la empresa tiene muy poca influencia en cómo los públicos perciben 
las actividades de la organización. Para que los stakeholders estén satisfechos, la clave radica en mantener una vigilancia constante de lo que acontece alrededor de la empresa e informarlo con la inmediatez y objetividad mayormente posible (Marston, 1999).

La reputación corporativa es frágil y valiosa. Influye en las preferencias de los consumidores, en donde invertir, así como en los posibles empleados cuando solicitan un puesto. "Evaluar y manejar la reputación de una empresa es tan importante como promover sus productos. La evaluación empieza cuando los líderes de la empresa dedican tiempo a raizar encuestas y entrevistas para averiguar lo que la gente piensa de la organización" (Baack, 2010, p. 359). Este proceso debe incluir los puntos de vista internos sobre la reputación de la empresa, así como las opiniones de personas ajenas a esta.

La responsabilidad social forma parte de la nueva cultura que está generando cambios en el ambiente corporativo. Se ha convertido en una opción estratégica para prácticas sociales y ambientales que contribuyen a la mejora de la calidad de vida para las próximas generaciones. Las empresas que creen en estos lineamentos deben actuar con coherencia e integridad. El departamento de relaciones públicas tiene la tarea de "asegurar que los públicos internos estén enterados de los esfuerzos de responsabilidad social de la corporación, informando al público de estas actividades, comunicando y transmitiendo los valores organizacionales, el cuidado ambiental, el desarrollo de relaciones con la comunidad, con los proveedores y clientes" (Rebeil, 2012, p. 195).

\subsection{Modelos de relaciones públicas}

Junto con la teoría situacional de los públicos, los cuatros modelos propuestos por Grunig, constituyen uno de los aportes fundamentales dentro de las relaciones públicas.

- Modelo agente de prensa: las relaciones públicas realizan una función persuasiva, propagandística y desinformativa. Los profesionales difunden información, a menudo incompleta y deformada, a sus clientes. Se trata de un modelo de comunicación unidireccional de la organización hacia sus públicos.

- Modelo de información pública: el fin de las relaciones públicas es la difusión de información no necesariamente con finalidad persuasiva. El profesionista actúa como si de un periodista integrado a la organización se tratara. El modelo de comunicación también es unidireccional.

- El modelo asimétrico bidireccional: tiene como finalidad e persuadir científicamente a los públicos, utilizando métodos y técnicas de las ciencias sociales para estudiar actitudes y comportamientos de los públicos, para que acepten el punto de vista de la organización y se comporten de manera que secunden sus decisiones. La comunicación es bidireccional, fluye al público y de desde este (retroalimentación)

- Modelo simétrico bidireccional: constituye el ideal de las relaciones públicas. Los publirrelacionistas que lo practican actúan como mediadores entre la organización y los públicos de su entorno. El fin es el entendimiento mutuo, 
un diálogo a que la organización y el público modifiquen sus actitudes y comportamiento después de la ejecución del programa de relaciones públicas (Xifra, 2003, pp. 53-56).

Diferentes estudios sobre comunicación se han interrogado acerca de las perspectivas más adecuadas para el analizar de las relaciones públicas contemporáneas. En los ochentas, algunos académicos sugirieron la teoría general de los sistemas, ya que la función relacional forman parte de un mecanismo sistémico (Xifra, 2003).

Figura 4: Estudiantes de nuevo ingreso del proceso de intercambio.

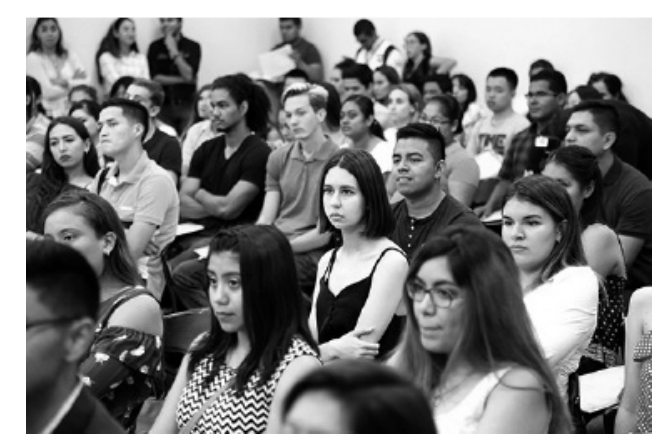

Fuente: UANL (2018d).

Conforme al modelo simétrico bidireccional, en cada semestre, la FCC de la UANL (en coordinación con la DIA) realiza eventos institucionales a modo de acompañamiento y asesoría a los estudiantes de intercambio (figura 4).

Figura 5: Testimonial de estudiante de la Universidad Veracruzana.

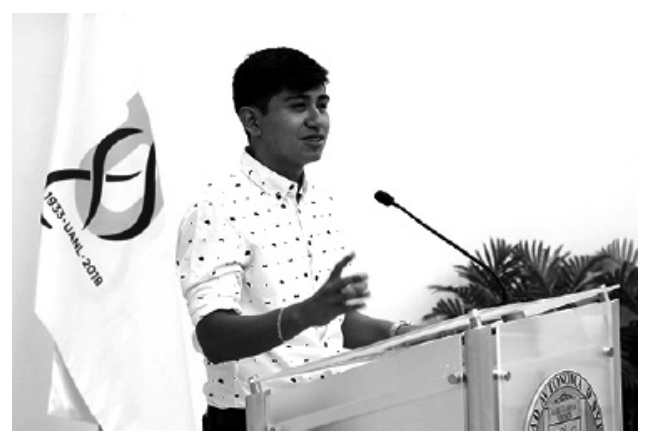

Fuente: UANL (2018d).

Del mismo modo, se ofrecen testimoniales de estudiantes quienes transcurrieron en el proceso de intercambio en semestres anteriores (figura 5). Asimismo, se organizan ceremonias de bienvenida a los estudiantes foráneos (figura 6), acompañados por los coordinadores de Facultades y directivos del DIA y de la UANL. 
Figura 6: Estudiantes de universidades nacionales e internacionales para cursar el semestre agosto-diciembre 2018.

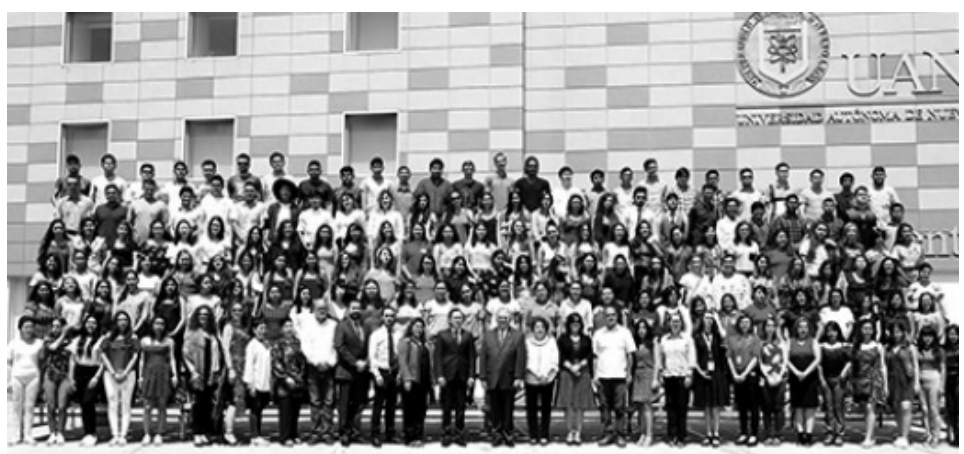

Fuente: UANL (2018d).

A fin de asegurar su integración a la universidad y a sus nuevos condiscípulos, los estudiantes extranjeros (figura 7) reciben apoyo para facilitarles las gestiones referidas a la homologación y registro de las unidades de aprendizaje cursadas en sus centros de estudios de origen.

Figura 7: Estudiantes sudamericanos de intercambio para el semestre agosto-diciembre 2018.

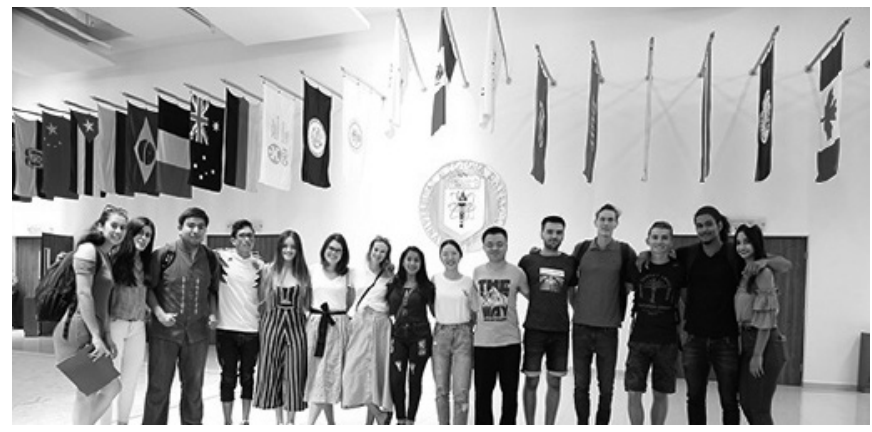

Fuente: UANL (2018d).

\section{Metodología}

La investigación se aborda desde el método cuantitativo-cualitativo, referidos a los estudios mixtos que evalúan el fenómeno o situación a investigar (Hernández-Sampieri \& Mendoza, 2018), con un alcance descriptivo y un diseño de investigación no experimental transeccional descriptivo. Se sigue la siguiente premisa de investigación: la identidad corporativa de la dependencia, propicia la singularización y distinción entre los miembros que la conforman. 
Se considera un universo de 3080 alumnos (que forman parte de la comunidad estudiantil), cuya muestra es de 342 sujetos a medir, obteniéndose un $95 \%$ de confianza y un margen de error del 5\%. La tipología de la muestra es aleatoria simple y su selección considera alumnos inscritos en la dependencia, pertenecientes a cualquiera de las tres licenciaturas (Comunicación, Periodismo Multimedia y Mercadotecnia y Gestión de la Imagen) registrados en cualquiera de los tres turnos.

Las técnicas seleccionadas para la recolección de datos fue la encuesta, desarrollada en un cuestionario de construcción propia que mide la categoría de identidad corporativa en siete signos de identidad (geográficos, arquitectónicos, de prestigio, de servicio, lingüísticos, iconográficos y tecnológicos) distribuidos en 16 ítems aplicados cara a cara. Se revisaron documentos institucionales en formatos impresos y digitales, así como fuentes bibliográficas de primera mano.

\section{Resultados}

Con respecto a los rangos de edad (figura 8), el 48\% de los encuestados tuvieron 20-21 años, el 36\% entre 17 y 19 años. Los encuestados mayores (22-25 años) representaron el $16 \%$, Estos valores representan la mayor participación de los estudiantes en la encuesta en los últimos semestres.

Figura 8: Datos poblacionales (edad).

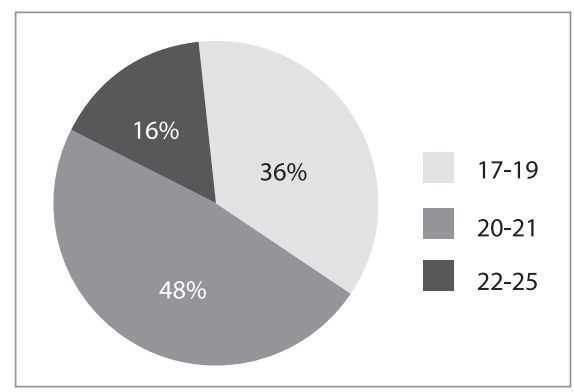

Fuente: Elaboración propia.

Con respecto al género (figura 9), los sujetos participantes de la muestra tienen casi el mismo valor porcentual y, por tanto, hay equidad en la emisión de su respuesta. Esto último es valioso, porque se podrá apreciar su punto de vista en las siguientes respuestas. 
Figura 9: Datos poblacionales (género).

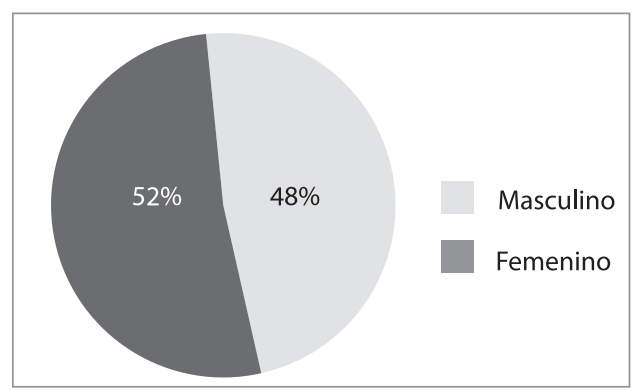

Fuente: Elaboración propia.

En la figura 10 se evidencia que la gran mayoría de los encuestados son solteros, que se ajusta al perfil promedio de los estudiantes en la UANL.

Figura 10: Datos poblacionales (estado civil).

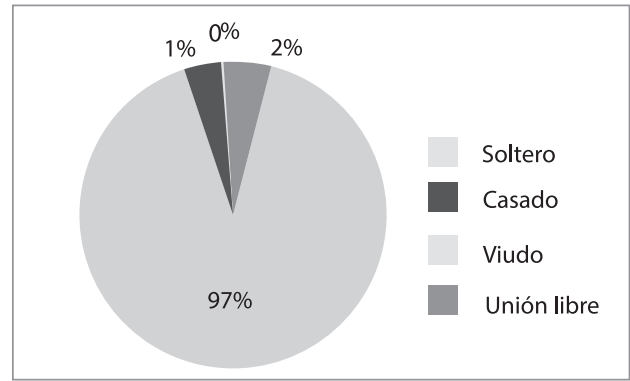

Fuente: Elaboración propia.

Con respecto al origen de los estudiantes, el 21\% provienen de Monterrey, el 21\% de Zuazua, el 11\% de Anáhuac y el 10\% de Guadalupe (figura 11).

Figura 11: Datos poblacionales (localidad).

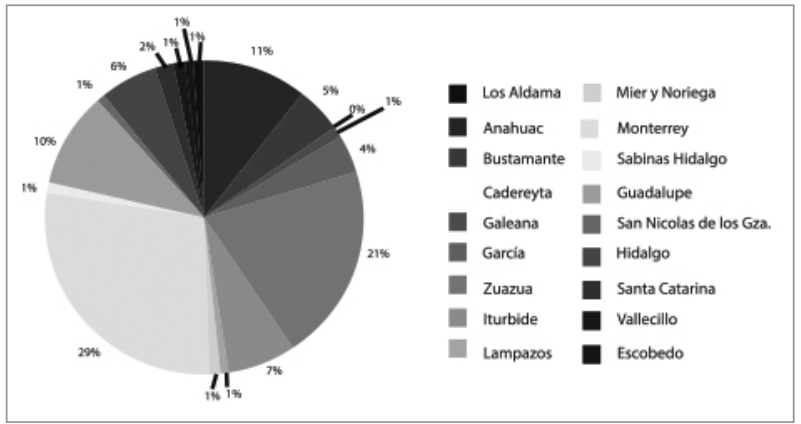

Fuente: Elaboración propia. 
La mayoría de los encuestados pertenecen a la carrera de Ciencias de la Comunicación, con un $83 \%$, (figura 12), el $15 \%$ a la de Mercadotecnia y Gestión de la Imagen y un 2\% a Periodismo Multimedia. Cabe destacar que las dos últimas carreras mencionadas son de reciente creación y aparecen en el quinto semestre.

Figura 12: Datos académicos (carrera).

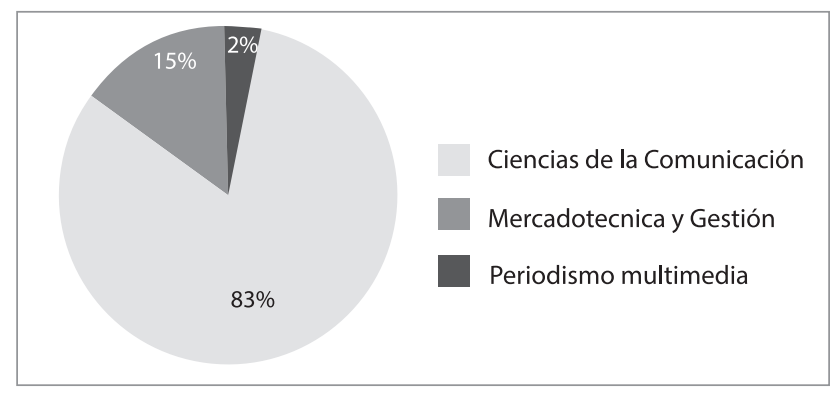

Fuente: Elaboración propia.

Ante la pregunta, “¿Consideras que las instalaciones de la FCC son adecuadas para el desempeño académico y le agregan valor a la identidad corporativa de la FCC?" (figura 13), el $47 \%$ de la muestra considera las instalaciones adecuadas para el desempeño académico; el 15\% muy de acuerdo; el 22\% ni de acuerdo ni en desacuerdo; el $13 \%$ en desacuerdo y el 3\% muy en desacuerdo. Así, el $62 \%$ emite una respuesta positiva por un amplio margen.

Figura 13: Categoría identidad (signo arquitectónico).

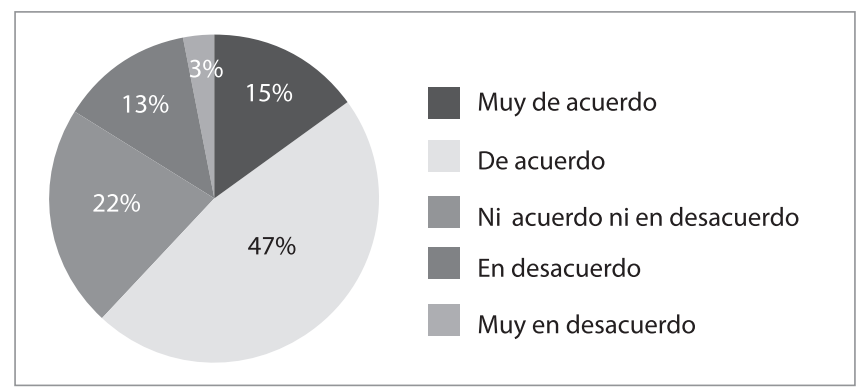

Fuente: Elaboración propia.

Ante la pregunta, "Consideras que la carrera de comunicación está bien posicionada académicamente y le confiere prestigio?" (figura 14), el 10\% de los encuestados se muestran muy de acuerdo; el 35\% de acuerdo; el 33\% ni de acuerdo ni en desacuerdo; el $17 \%$ en desacuerdo y el $5 \%$ totalmente en desacuerdo. En este punto se evidencian respuestas divididas: un $45 \%$ de la muestra piensa que la carrera está desfavorablemente ubicada, contra un 55\% que la consideran adecuadamente posicionada en el contexto académico nacional. 
Figura 14: Categoría identidad (signo de prestigio).

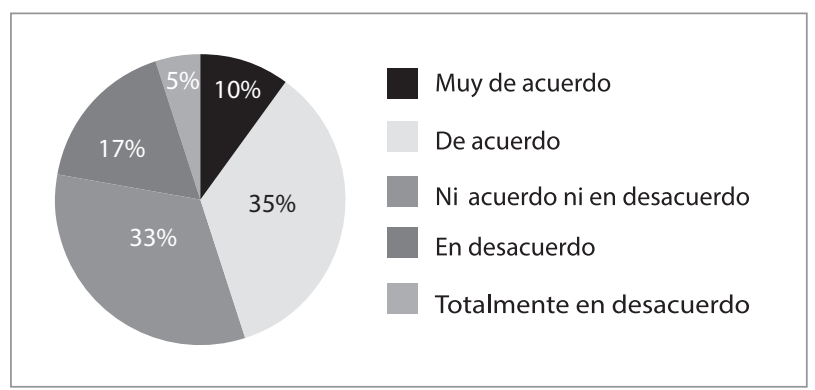

Fuente: Elaboración propia.

Ante la pregunta, “¿Consideras que las carreras en formación de mercadotecnia y gestión de la imagen y periodismo multimedia cumplen las expectativas de los estudiantes?" (figura 15), el 12\% de los encuestados están muy de acuerdo; el 35\% de acuerdo; el 34\% ni de acuerdo ni en desacuerdo; el $18 \%$ en desacuerdo y un $1 \%$ muy en desacuerdo. La inclinación mayoritaria es de un $53 \%$ con una tendencia negativa, contra un $47 \%$ que consideran cumplidas sus expectativas.

Figura 15: Categoría identidad (signo de servicio).

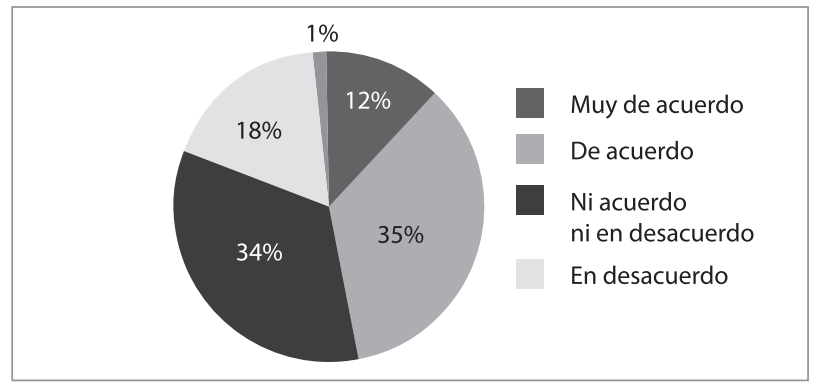

Fuente: Elaboración propia.

Ante la pregunta, “¿Conoces el lema de la FCC?” (figura 16), el 38\% de la muestra aseguró que el lema era "Comunicación somos todos"; un 7\% "Pasión por comunicación"; el 39\% "In principium Erat Verbum"; el 10\% "Alere Flammam Veritatis" y el 6\% "Por un futuro profesional". El lema correcto de la FCC es "In principium Erat Verbum", mientras que los otros restantes, tales como "Comunicación somos todos", correspondió a una campaña de integración. "Pasión por comunicación" y "Por un futuro profesional" formaron parte de la campaña para elegir un nuevo director. 
Figura 16: Categoría identidad (signo de lingüística).

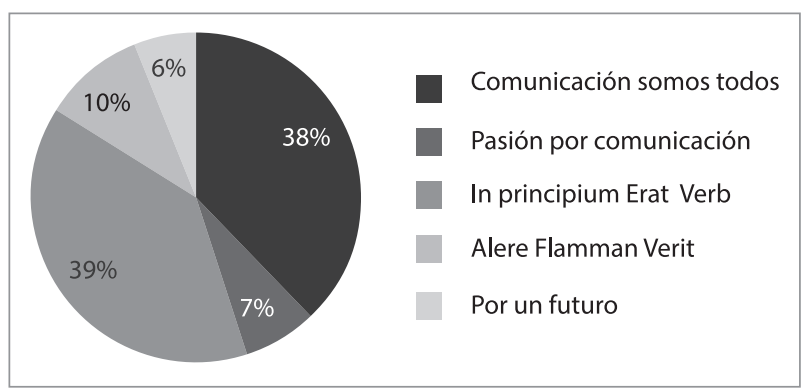

Fuente: Elaboración propia.

Ante la pregunta, "Consideras que el lema de la FCC es pertinente a la carrera y le agrega valor de identidad?" (figura 17), el 17\% de los encuestados están muy de acuerdo con la pertinencia del lema; el 39\% sólo de acuerdo; un 25\% ni de acuerdo ni en desacuerdo; el $15 \%$ en desacuerdo y un $4 \%$ muy en desacuerdo. Se percibe una tendencia positiva del $56 \%$, contra un $44 \%$ negativa, entreviéndose el deseo de cambiar el lema de la FCC.

Figura 17: Categoría identidad (signo de lingüística).

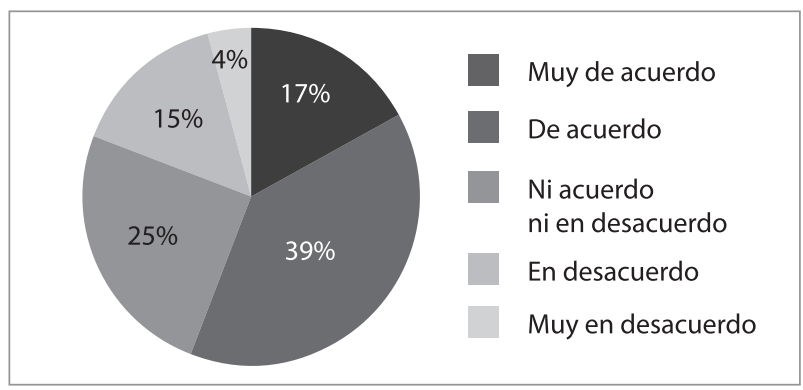

Fuente: Elaboración propia.

Ante la pregunta “¿Consideras que la ubicación de la FCC se encuentra en un lugar adecuado rodeada del contexto natural de cerros y plantas endémicas, favoreciendo la identidad?" (figura 18), el 17\% está muy de acuerdo; el 37\% sólo de acuerdo; el 22\% ni de acuerdo ni en desacuerdo y un 6\% muy en desacuerdo. La tendencia indica que mayoría encuentran adecuada la ubicación geográfica de la FCC. 
Figura 18: Categoría identidad (signo geográfico-ubicación).

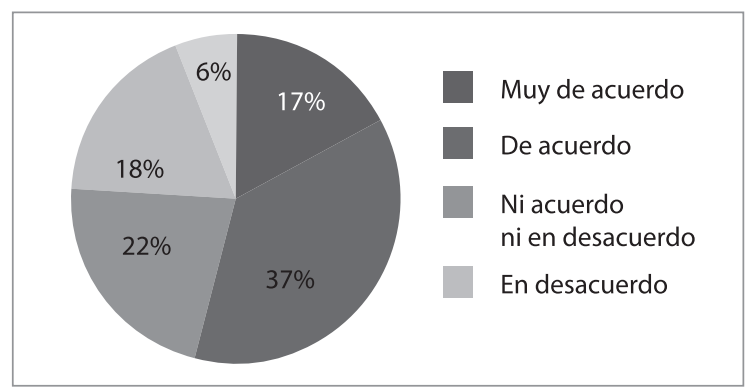

Fuente: Elaboración propia.

Ante la pregunta, “¿Conoces el significado del imagotipo de la FCC?” (figura 19), el 12\% de la población admite conocer bastante su significado; el 29\% parcialmente; el 25\% algo; el $24 \%$ un poco y el $10 \%$ nada. Si bien la tendencia es positiva, hay que tomar en cuenta el porcentaje de los estudiantes que carecen de la información del imagotipo de la FCC.

Figura 19: Categoría identidad (signo iconográfico).

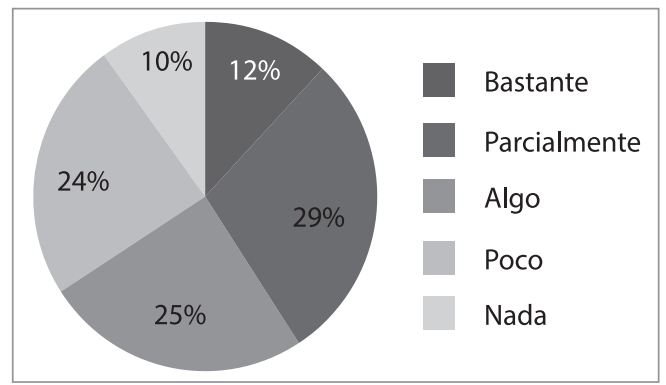

Fuente: Elaboración propia.

Ante la pregunta: “¿Crees que la mascota de la FCC le proporciona identidad?” (figura 20), el 28\% de la muestra dice estar muy de acuerdo; el 35\% de acuerdo; el 20\% ni de acuerdo ni en desacuerdo; el 14\% en desacuerdo y el 3\% muy en desacuerdo. 
Figura 20: Categoría identidad (signo iconográfico).

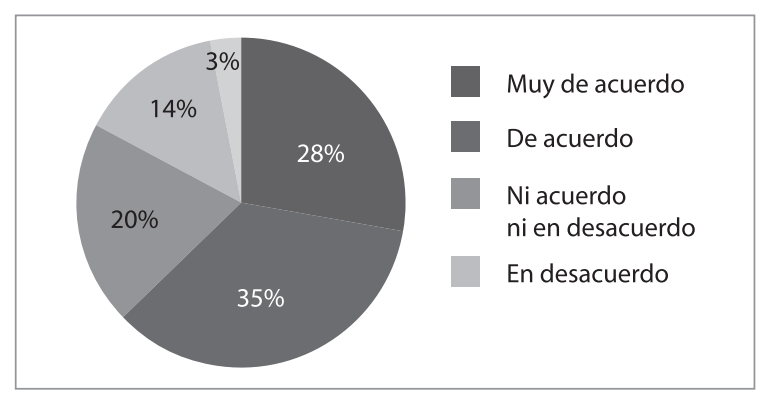

Fuente: Elaboración propia.

Ante la pregunta: “Consideras que las tecnologías académicas son adecuadas para el proceso de enseñanza-aprendizaje y estas le agregan valor a la identidad corporativa de la FCC?" (figura 21), el 17\% de los estudiantes consideran estar muy de acuerdo; el 40\% sólo de acuerdo; el 20\% ni de acuerdo ni en desacuerdo; el 17\% en desacuerdo y el $6 \%$ muy en desacuerdo.

Figura 21: Categoría identidad (signo de tecnología).

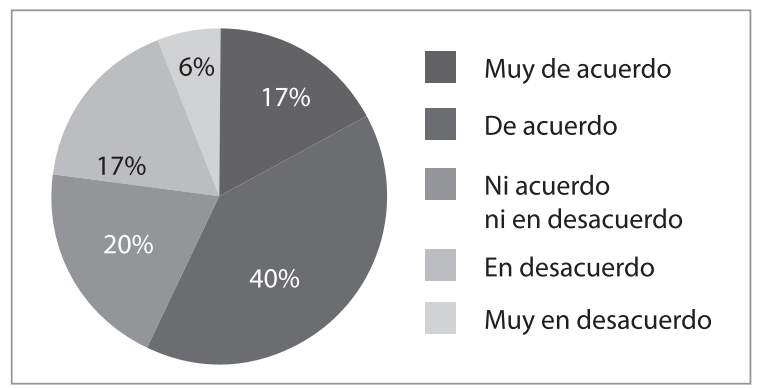

Fuente: Elaboración propia.

Ante la pregunta: “¿Consideras que el profesorado está capacitado para impartir clases en las diferentes unidades de aprendizaje y su desempeño le agrega valor a la identidad de la FCC?" (figura 22), la muestra considera que un 11\% está bastantemente capacitado; un 36\% parcialmente; un $29 \%$ algo; un $17 \%$ poco y un $7 \%$ nada. La tendencia de los resultados es negativa con un 53\%, evidenciándose que el profesorado no está capacitado para impartir determinadas unidades académicas, lo que resta valor a la identidad de la FCC. 
Figura 22: Categoría identidad (signo de servicio).

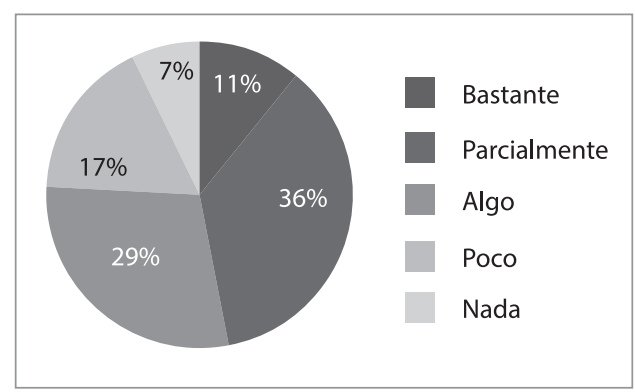

Fuente: Elaboración propia.

Por último, con respecto al nivel de identidad corporativa que tienen los profesores de la FCC (figura 23), un 16\% de los encuestados lo califica como excelente; un 39\% buena; un $31 \%$ sólo regular; un $9 \%$ mala y un 5\% pésima.

Figura 23: Categoría identidad (signo de servicio).

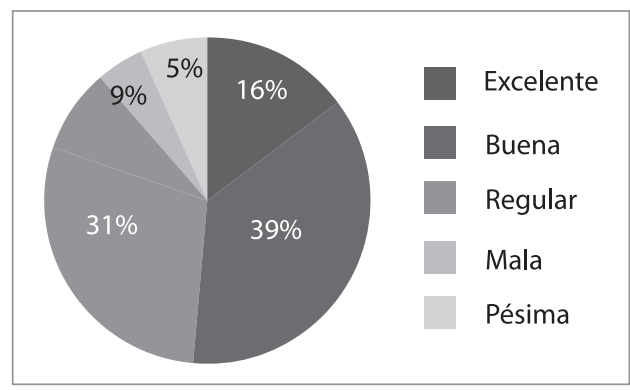

Fuente: Elaboración propia.

En suma, la tendencia es ligeramente positiva, con un $55 \%$ frente a un $45 \%$ negativa. Estos resultados determinan que existen elementos negativos que deben analizarse con más detenimiento, a fin de optimizar el grado de identidad corporativa entre el profesorado.

\section{Conclusiones}

Mientras no surgen problemas en lo referido a los signos de identidad geográfica y arquitectónica (componentes positivos que agregan valor a la identidad), aspectos relacionados con la identidad de prestigio (45\%) y de servicio (53\%) muestran considerables tendencias negativas, sobre todo el último, ya que los estudiantes consideran que la realización de trámites y servicios administrativos (que gestionan al inicio de semestre), no es eficiente. 
Si bien los encuestados están familiarizados con el lema de la FCC (y lo consideran pertinente) y la mayoría desconoce el significado del imagotipo. Con respecto a los signos de identidad tecnológica, el equipo y mobiliario (que forma parte del sistema tecnológico) son los adecuados para el proceso de enseñanza-aprendizaje y le suma valor a la identidad de la FCC, complementando así a una infraestructura que aporta conectividad a toda la comunidad universitaria.

Los encuestados subrayan que la FCC goza de un buen posicionamiento en el ámbito académico y que las carreras de nueva creación como Mercadotecnia y Gestión de la Imagen, Periodismo Multimedia y Ciencias de la Comunicación, hasta ahora, han cumplido sus expectativas y coadyuvan a una buena identidad de la dependencia. Con la finalidad de mantener positivamente la identidad corporativa y el prestigio en los públicos internos, los resultados sugieren que se capacite a los profesores de reciente ingreso para actualizar y clarificar los contenidos de sus unidades académicas.

Este tipo de prácticas de retroalimentación continua hace que el modelo de relaciones públicas que realiza la FCC es el simétrico-bidireccional, en términos de la teoría general de sistemas, es decir, homeostática.

\section{Referencias}

Benjamín, E. \& Fincowsky, F. (2014). Organización de empresas. México, DF: McGraw Hill.

Baack, C. (2010). Publicidad, promoción y comunicación integral en marketing. México, DF: Pearson.

Chiavenato, I. (2009). Comportamiento organizacional. México, DF: McGraw-Hill.

Castillo, H. \& Camacho, M. (2017). Comunicación y liderazgo decisional. México, DF: Éxodo.

Costa, J. (2011). Identidad corporativa. México, DF: Trillas.

Fernández, C. (2014). La comunicación en las organizaciones. México, DF: Trillas.

Fernández, C. \& Galguera, L. (2008). La comunicación humana en el mundo contemporáneo. México, DF: McGraw-Hill.

González, C. (2015). Principios básicos de comunicación. México, DF: Trillas.

Hernández-Sampieri, R. \& Mendoza, C. (2018). Metodología de la investigación. Las rutas cuantitativa, cualitativa y mixta. México, DF: Mc-Graw Hill.

Koontz, H., Weihrich, H. \& Cannice, M. (2012). Administración. México, DF: McGraw-Hill. 
Limón, M. (2008). Imagen corporativa. Estrategia organizacional de comunicación global. México, DF: Trillas.

López Lara, Y. (2010). Propuesta de bases metodológicas para la gestión de comunicación interna dirigida al cambio sustentable en instituciones de educación superior Caso: Universidad Autónoma de Nuevo León (tesis doctoral). Universidad de la Habana, Cuba.

Martínez de Velazco, A. \& Nosnik, A. (2008). Comunicación organizacional práctica. México, DF: Trillas.

Marston, J. (1999). Relaciones Públicas modernas. México, DF: McGraw-Hill.

Mello, F. (2009). Desarrollo organizacional, enfoque integral. México, DF: Limusa.

Paoli, J. (1986). Comunicación e información. Perspectivas teóricas. México, DF: Trillas.

Real Academia Española, RAE (2018). Definición de identidad. Recuperado de http:// dle.rae.es/srv/search?m=30\&w=identidad

Rebeil, M. (2012). Responsabilidad social organizacional. México, DF: Trillas.

Ríos, J. (2006). Relaciones Públicas. Su administración en las organizaciones. México, DF: Trillas.

Robbins, S. \& Judge, T. (2017). Comportamiento organizacional. México, DF: Pearson.

Universidad Autónoma de Nuevo León, UANL (2018a). Centro de Internalización. Recuperado de http://internacional.uanl.mx/centro-de-internacionalizacion-2/

Universidad Autónoma de Nuevo León, UANL (2018b). Visión 2020. Recuperado de http://www.uanl.mx/utilerias/vision2020.pdf

Universidad Autónoma de Nuevo León, UANL (2018c). Facultad de Ciencias de la Comunicación. Recuperado de www.comunicacion.uanl.mx

Universidad Autónoma de Nuevo León, UANL (2018d). "Estudiantes del programa de intercambio. Semestre agosto-diciembre 2018”. Dirección de Comunicación Institucional y Relaciones Públicas. Departamento de Prensa.

Xifra, J. (2003). Teoría y estructura de las relaciones públicas. Madrid: McGraw-Hill.

Wilcox, D., Cameron G. \& Xifra, J. (2012). Las Relaciones Públicas. Estrategias y tácticas. México, DF: Pearson. 